\title{
NIST REFERENCE SPECTRAL RESPONSIVITY SCALES FOR IMPROVING THE SI CANDELA AND KELVIN
}

\author{
G. P. Eppeldauer, S.W. Brown, K. R. Lykke, C. C. Miller, Y. Ohno, and H.W. Yoon \\ National Institute of Standards and Technology \\ 100 Bureau Drive, Gaithersburg, Maryland, USA
}

\begin{abstract}
$\underline{\text { Abstract }}$
Both SI units, the candela and the kelvin were originally derived from standard blackbody sources. Recently, instead of source-based calibrations, they both have been realized with optical detector standards $[1,2]$. Recent decrease of the measurement uncertainties in detector-based calibrations resulted in improved uncertainties in these SI units.
\end{abstract}

\section{$\underline{\text { Introduction }}$}

Primary-standard cryogenic radiometers can measure optical power with a relative expanded uncertainty of $0.02 \%(k=2)$ [3]. When the optical power scale is properly transferred to spectral responsivity scales, the responsivity uncertainties will have only a minimal increase. These scale transfers need welldesigned transfer standard detectors and a spectral responsivity calibration facility that produces decreased responsivity uncertainties compared to traditional monochromator-based facilities. The recently developed detector transfer-standards and the decreased uncertainty spectral responsivity scale transfers that make the improvement of the candela and kelvin possible are discussed here.

\section{$\underline{\text { Irradiance measuring transfer-standard }}$}

New-generation transfer-standard silicon trapdetectors have been developed [4] that can be calibrated against the primary standards with a minimal increase in the uncertainty of radiant power responsivity. These trap-detectors, when equipped with a precision aperture of known area, are used as irradiance-measuring transfer standards with a measurement uncertainty of $0.06 \%(k=2)$. This irradiance responsivity reference scale is then transferred to newly developed filter-detector transfer-standards.

\section{Spectral responsivity calibration facility}

Simultaneously to the development of the detector transfer standards, a reference facility for Spectral Irradiance and Radiance Responsivity Calibrations using Uniform Sources (SIRCUS) has been developed at NIST [5]. Irradiance- and radiancemeasuring filter-detector transfer-standards such as illuminance-measuring photometers and radiancemeasuring spectrally-selective radiometers can be calibrated against the irradiance-measuring transfer standard trap-detectors with the smallest possible increase in the responsivity uncertainty. These filterdetectors are directly used to realize the above two SI units.

\section{$\underline{\text { The Illuminance-Unit }}$}

The present NIST illuminance unit, maintained on a group of eight photometers, has a relative expanded uncertainty of $0.39 \%(k=2)$ [1]. The dominant uncertainties (originating from the converging beam geometry, the small spot size on the photometers, wavelength errors, aperture area measurements error, and the spatial non-uniformity of detector and filter combination) are related to the traditional lamp/monochromator spectral comparator facility (SCF) used to calibrate the eight photometers [1]. Calibrating these photometers with the tunable laser sources of the SIRCUS would increase the calibration uncertainty because of the interference fringes in their output signals. New generation, fringe-free transfer-standard photometers have been developed with optimized optical, photometric, and electronic characteristics. An improved photometric scale is being realized at SIRCUS using the new transfer standard photometers [6]. The eight existing photometers or other standards-quality photometers can be calibrated against the new transfer standards by measuring the same illuminance from a $2856 \mathrm{~K}$ color temperature lamp. As a result of the lower uncertainty of the spectral irradiance responsivity and the yearly cleaning of the filter's front surfaces, the uncertainty of routine illuminance meter calibrations can be reduced by at least a factor of two.

\section{$\underline{\text { Radiance temperature measurements }}$}

At present, in the International Temperature Scale of 1990 (ITS-90) [2], temperatures above the freezing point of silver are determined with radiation thermometers calibrated using spectral radiance ratios to a freezing temperature blackbody and utilizing the Planck's radiation law. Unfortunately, the temperature uncertainties increase with the square of the temperature ratios resulting in large uncertainties at high temperatures. The uncertainties can be 
decreased when the radiation thermometers are calibrated for radiance responsivity against a lowuncertainty irradiance-measuring transfer-standard trap-detector. It was shown [7] that the spatial and angular uniformity of the integrating spheres used in the irradiance-to-radiance transfer can affect the radiance responsivities of the radiation thermometers resulting in increased temperature uncertainties. With the decreased uncertainty of the spectral radiance responsivity calibrations, a blackbody temperature can be directly determined from a radiance measurement without the use of any fixed points. Using a $305 \mathrm{~mm}$ diameter sphere and an irradiance mode trap-detector both with uniform spatial and angular characteristics, the obtained Gold-point temperature was $1337.38 \mathrm{~K}, 50 \mathrm{mK}$ higher than the ITS-90. The relative expanded temperature uncertainty was $+/-47 \mathrm{mK}(k=2)$.

\section{$\underline{\text { References }}$}

[1] C. L. Cromer, G. Eppeldauer, J. E. Hardis, T. C. Larason, Y. Ohno, and A. C. Parr, "The NIST detector-based luminous intensity scale," J. Res. Natl. Inst. Stand. Technol., vol. 101, pp. 109132, 1996.

[2] H. W. Yoon, D. W. Allen, C. E. Gibson, M. Litorja, R. D. Saunders, S. W. Brown, G. P. Eppeldauer, and K. R. Lykke, "Thermodynamictemperature determinations of the $\mathrm{Ag}$ and $\mathrm{Au}$ freezing temperatures using a detector-based radiation thermometer," Appl. Opt., vol. 46, no. 15, pp. 2870-2880, 2007.

[3] J. M. Houston and J. P. Rice, "NIST reference cryogenic radiometer designed for versatile performance," Metrologia, vol. 43, pp. S31-S35, 2006.

[4] G. P. Eppeldauer and D. C. Lynch, "Optomechanical and electronic design of a tunnel-trap Si-radiometer," J. Res. Natl. Inst. Stand. Technol., vol. 105, pp. 813-828, 2000.

[5] S. W. Brown, G. P. Eppeldauer, and K. R. Lykke, "Facility for spectral irradiance and radiance responsivity calibrations using uniform sources," Appl. Opt., vol. 45, no. 32, pp. 82188237, 2006.

[6] G. P. Eppeldauer, C. C. Miller, and Y. Ohno, "New photometer standards for a low uncertainty illuminance scale realization," The $26^{\text {th }}$ Session of the CIE, July 4-11, 2007, Beijing, China, in Proceedings volume 1, pp. D2-99 to D2-102.

[7] H. W. Yoon, S. W. Brown, G. P. Eppeldauer, and K. R. Lykke, "Properties of integrating spheres used in detector-based temperature realizations," LNE Meeting, Paris, France, 2006. 